\section{FULMINANT AMOEBIC COLITIS CAUSING CAECAL GANGRENE WITH RUPTURED LIVER ABSCESS}

KEY WORDS: Amoebic abscess, caecal gangrene,liver abscess,percutaneous drainage,laparatomy.

\section{Dr.Suganth.A*}

\section{Dr.Vimala.G}

Post graduate ,Institute of General surgery ,Madras Medical college and Rajiv Gandhi Government General Hospital, Chennai-3*Corresponding Author

Assistant professor,Institute of General surgery ,Madras Medical college and Rajiv Gandhi Government General Hospital,Chennai-3

\title{
Dr.Kannan.R
} Gandhi Government General Hospital,Chennai-3

Liver abscess is the most common extra intestinal manifestation in patients with amoebiasis.Amoebic abscess can present in paracaecal region and liver.Synchronous presence of caecal gangrene with ruptured liver abscess into peritoneum is rare.It has a high rate of morbidity and mortality especially in malnourished patients.Here we present a case of 66 years old male who presented to casualty with complaints of abdomen pain with abdomen distension.Radiological investigations showed ruptured liver abscess.Inview of peritonitis,patient was shifted to emergency theatre for laparotomy.Intraoperative findings were ruptured liver abscess with Caecum found to be sloughed out and gangrenous.Removal of Caecum with limited resection of ascending colon and distal ileum done and bowel exteriorised.Post operatively patient started on oral diet on second day and discharged on seventh day .Patient was readmitted for abdomen pain and percutaneous drainage was done for residual liver abscess.Prompt suspicion of caecal pathology should always be made in patients with ruptured liver abscess and early intervention is needed to prevent mortality.

\section{INTRODUCTION:}

Liver abscess is the most common extraintestinal manifestation in amoebiasis.Amoebic abscess can present in paracaecal region and liver.Synchronous presence of caecal gangrene with ruptured liver abscess into peritoneum is rare.It has a high rate of morbidity and mortality in malnourished patients.

\section{CASE REPORT}

66 years old male patient came to casualty with complaints of abdomen pain for 5 days and abdomen distension for 2days.He had History of intermittent fever and constipation. On examination, he has abdomen tenderness and guarding in right upper quadrant and right iliac fossa.Pulse rate:112/min.Bp:110/70mmhg.

USG abdomen showed well defined hypoechoic lesion of size $8 * 7 \mathrm{~cm}$ in segment 7 of liver. $1 * 1 \mathrm{~cm}$ in segment 8 of liver with subdiaphragmatic collection

CECT abdomen with iv contrast showed multiple well defined hypodense lesions with peripheral rim enhancement in segment 7,8 of size $11.8 * 9.2 \mathrm{~cm}$ with multiple air pockets.Evidence of large subdiaphragmatic collection noted extending into subhepatic,right paracolicand right iliac fossa with large air pockets.Possibility of right lobe liver abscess with rupture

Inview of peritonitis ,emergency laparatomy done.400ml purulent peritoneal fluid aspirated.Omentum found adherent to caecum.Caecum found to be perforated,sloughed out and gangrenous.Flakes seen over the surface of liver.

Proceeded with limited resection of caecum with distal ileum and remaining ends of bowel brought out as ostomy.Patient was started on oral diet on second day and discharged on 7th day.Patient was readmitted for abdomen pain and percutaneous drainage done for residual liver abscess.

\section{DISCUSSION:}

The most common etiology of liver abscess is secondary to another intraabdominal infection like diverticulitis, appendicitis. Amoebiasis is common in tropical countries like India where Entamoeba histolytica is endemic.Mostly affects men and age group of 20 to 40 yrs.Alcohol intake and immunocompromised state are one of the risk factors for amoebic infection.

Entamoeba histolytica is a protozoan which exists in 2 forms namely tropozoite and cysts.Mode of entry is through fecooral route.After ingestion of contaminated vegetables and fruits with amoeba,the cysts reach stomach and small intestine.In intestine ,tropozoites are released and passed on to colon.By portal venous system the tropozoites reach liver where by enzymatic cellular hydrolysis they cause hepatic necrosis.

Liquefaction necrosis results in cavity being filled with blood and liquefied liver tissue and the fluid resembles anchovy sauce.Glisson capsule is resistant to amoebic hydrolysis. Amoebic liver abscess also affects mainly right lobe of liver.

Synchronous hepatic abscess is seen in one third of patients with amoebic colitis.In patients with amoebic liver abscess they have mild to moderate leucocytosis.The most common LFT abnormality is an elevated PT and INR.Since amoebae are not seen in stools in $70 \%$ of patients ,antiamoebic antibodies are measured by enzyme immunoassays.Recent studies have found the measurement of Entamoeba lecithin antigen in serum and liver pus to be sensitive.Ultrasound findings of liver abscess are the cavity seen as hypoechoic and nonhomogeneous. Computed tomography of abdomen is more sensitive than ultrasound .Rim enhancement noted in pyogenic liver abscess.Gallium scanning or technetium Tc $99 \mathrm{~m}$ liver scans help to differentiate between amoebic and pyogenic abscess.Amoebic abscesses donot contain leucocytes and so they don't light up on the scans.

Mainstay of treatment is administration of metronidazole $750 \mathrm{mg}$ three times daily for 10days for amoebic liver abscess. Other drugs that can also be used are emetine hydrochloride and chloroquine. Intaluminal agent paramomycin also given to eradicate intestinal colonization. Aspiration of abscess recommended if there is diagnostic uncertainity or failure to respond to metronidazole in 3 to 5days or if there is risk of rupture of abscess

The most frequent complication of amoebic liver abcess is 
rupture into peritoneum, pleural cavity or pericardium.The mortality rate of patients with amoebic liver abscess is $5 \%$.But for a ruptured liver abscess it ranges from $6 \%$ to $50 \%$.

\section{Conclusion}

Prompt suspicion of caecal pathology should always be made with ruptured liver abscess and early intervention needed to prevent mortality.

\section{CT PICS OF RUPTURED LIVER ABSCESS WITH SUBDIAPHRAGMATIC COLLECTION}

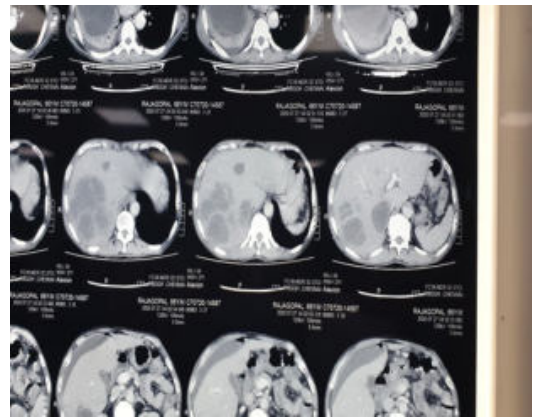

Picture 1.

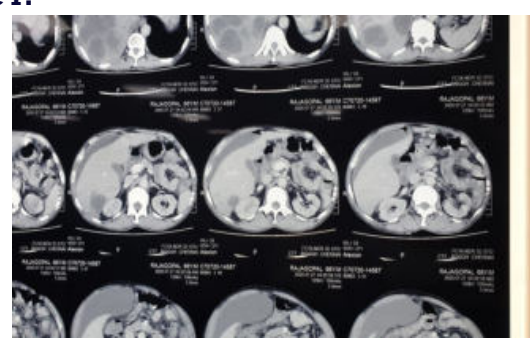

Picture 2.

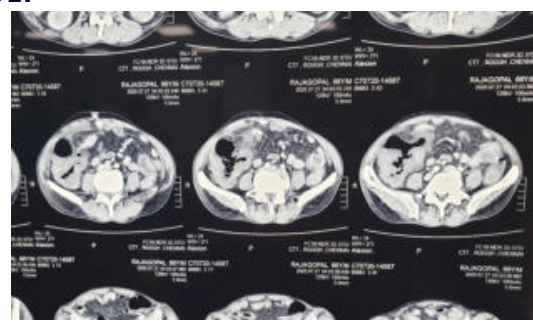

Picture 3.

\section{SPECIMEN OF RESECTED GANGRENOUS CAECUM WITH DISTAL ILEUM}

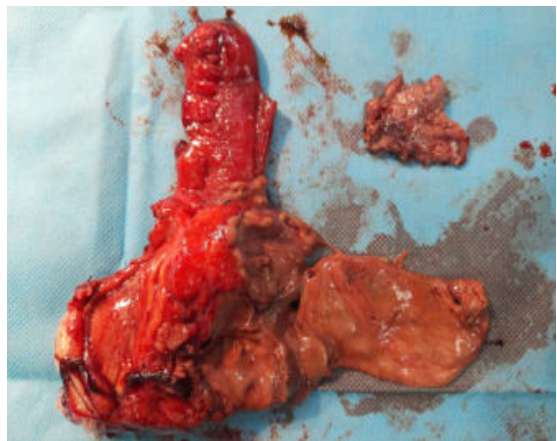

\section{References:}

1. Tanwar, Raman \& Jain, Sudhir. (2013). Amoebic cecal perforation following fulminant colitis with amoebic appendicitis and ruptured liver abscess: $A$ rare presentation. Annals of Tropical Medicine and Public Health. 6.367-368. 10.4103/1755-6783.121014.

2. Mohd Yasir Beg, Lovenish Bains, Ratnesh Mahajan, Pawan Lal, Sharmana Choudhury, N. Pritesh Kumar, Eva Wilse C. Momin, Veer Pal, "Fulminant Necrotising Amoebic Colitis of Whole of Large Bowel: A Rare Complication of a Common Infectious Disease", Case Reports in Infectious Diseases, vol. 2020, Article ID 8845263,6 pages, 2020.https://doi.org/10.1155/2020/8845263

3. P Karthick. Synchronous Ruptured Amoebic Liver Abscess With Caecal Perforation - A Rare Case Presentation. The Internet Journal of Surgery. 2010 Volume 27 Number 2 